\title{
Hipotensión ortostática: una manifestación sugerente de feocromocitoma
}

\author{
Rodrigo Tagle V, Pamela Acosta V, G loria Valdés S.
}

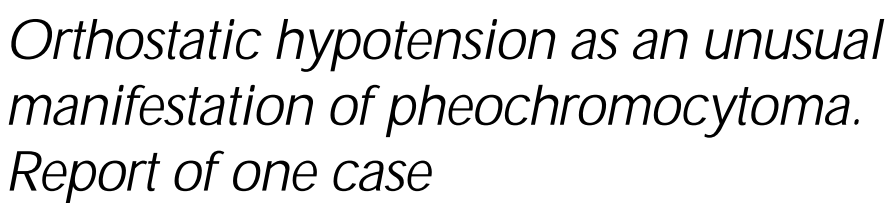

Pheochromocytoma, though an uncommon cause of hipertension, can be a lethal condition. Because of this it is mandatory to diagnose it or rule it out in presence of suggestive symptoms. Typical symptoms are palpitations, sweating, severe headaches and hypertension. However, there are other suggestive symptoms of this dangerous endocrine entity, one of which is the orthostatic hypotension. We report the case of a 65 years old female patient with long standing hypertension in whom the pheochromocytoma was suspected after episodes of orthostatic hypotension. Although this manifestation was described almost fifty years ago, its frequency and pathophysiology has not yet been well established and fully elucidated. Moreover, it has meaningful implications in relation to preoperatory management and the timing of surgery (Rev Méd Chile 2003; 131: 1429-33).

(Key Words: Hypotension; Orthostatic; Pheochromocytoma)

Recibido el 20 de mayo, 2003. Aceptado el 16 de septiembre, 2003.

Departamento de Nefrología, Pontificia Universidad Católica de Chile.

$\mathrm{E}^{\mathrm{I}}$ feocromocitoma es un tumor de las células cromafines, que puede localizarse en los territorios derivados de la cresta neural o en el trayecto que estas células realizan hasta su localización definitiva. Estos tumores se ubican en $90 \%$ en la región abdominal, particularmente en las glándulas adrenales y órgano de Zuckerkandl. Pueden causar una amplia variedad de síntomas, dado su capacidad de secretar catecolaminas, particularmente noradrenalina y adrenalina, en cantidades variables e intermitentes ${ }^{1}$. Aunque son bien conocidas sus manifestaciones clásicas tales como hipertensión

Correspondencia a: Rodrigo Tagle. Departamento de Nefrología. Pontificia Universidad Católica de Chile. Marcoleta 354, Santiago. Fono: 3543715. Fax: 6397377.

E mail: rtagle@med.puc.cl arterial paroxística o permanente, asociada a la tríada de cefalea, sudoración y palpitaciones, comúnmente no se presenta inicialmente como tal ${ }^{2}$. La hipotensión ortostática es una manifestación altamente sugerente de feocromocitoma en un paciente hipertenso, que tiene interesantes connotaciones fisiopatológicas y terapéuticas ${ }^{3,4}$.

Presentamos el caso de una paciente portadora de feocromocitoma, sospechado a raíz de hipotensión ortostática.

\section{CASO CLÍNICO}

Mujer de 65 años con hipertensión arterial desde hacía 15 años e hipotimidismo, en tratamiento con atenolol $50 \mathrm{mg} /$ día y levotiroxina $100 \mathrm{mcg} /$ día. 
Un mes previo a su ingreso, consultó por cefalea frontal frecuente, palpitaciones, disnea y labilidad emocional. Se diagnosticó síndrome depresivo y se inició terapia con imipramina $25 \mathrm{mg} /$ $12 \mathrm{~h}$. Posteriormente, los síntomas se agudizaron, particularmente las palpitaciones aumentaron en intensidad y frecuencia. El estudio cardiológico reveló sólo taquicardia sinusal y quedó en tratamiento con carvedilol $25 \mathrm{mg} /$ día, enalapril $10 \mathrm{mg} /$ día e hidroclorotiazida $25 \mathrm{mg} /$ día.

Persistió sintomática e ingresó por cefalea frontal intensa que aumentaba al ponerse de pie y disminuía con el decúbito, asociada a mareos y náuseas. La evaluación neurológica clínica y por imágenes resultó normal. Al tercer día de hospitalización presentó, al incorporarse de la cama, súbita hipotensión de $70 / 30 \mathrm{mmHg}$ y taquicardia sinusal de 160 por min, piel fría, sudoración profusa y piloerección; estos episodios se repitieron y alternaban con presiones arteriales de hasta 200/110 mmHg en decúbito supino.

Ante la sospecha de feocromocitoma, se inició infusión de cristaloides y se suspendieron los fármacos especialmente imipramina, carvedilol e hidroclorotiazida, por la interferencia en la interpretación y medición de metanefrinas urinarias y catecolaminas plasmáticas. Estas últimas se midieron en reposo y durante una crisis hipertensiva (Tablas 1 y 2).

Se realizó TAC de abdomen y pelvis sin contraste, y resonancia nuclear magnética que confirmaron el diagnóstico, detectando una masa de 30 por $35 \mathrm{~mm}$ en la glándula suprarrenal

Tabla 1. C atecolaminas Plasmáticas

\begin{tabular}{|lrrr|}
\hline & \multicolumn{1}{c}{ Basal } & \multicolumn{1}{c}{ Crisis } & Valores normales \\
\hline Adrenalina & $181 \mathrm{pg} / \mathrm{ml}$ & $209 \mathrm{pg} / \mathrm{ml}$ & $20-82 \mathrm{pg} / \mathrm{ml}$ \\
Noradrenalina & $2.486 \mathrm{pg} / \mathrm{ml}$ & $3.127 \mathrm{pg} / \mathrm{ml}$ & $41-773 \mathrm{pg} / \mathrm{ml}$ \\
\hline
\end{tabular}

Tabla 2. M etanefrinas urinarias $24 \mathrm{~h}$

\begin{tabular}{|lrr|}
\hline & \multicolumn{1}{c}{ Valor } & Valores normales \\
\hline Metanefrinas & $982 \mathrm{ug} / 24 \mathrm{~h}$ & $52-341 \mathrm{ug} / 24 \mathrm{~h}$ \\
Normetanefrinas & $2.920 \mathrm{ug} / 24 \mathrm{~h}$ & $88-444 \mathrm{ug} / 24 \mathrm{~h}$ \\
\hline
\end{tabular}

izquierda de estructura heterogénea con signo del anillo y refuerzo de señal en T2, compatibles con feocromocitoma (Figuras 1 y 2). El cintigrama con metaiodobencilguanidina (MIBG) confirmó la captación en la masa descrita y no mostró metástasis.

Se preparó para la intervención quirúrgica con aporte oral de sal y solución fisiológica, y bloqueo $\alpha$ y $\beta$-adrenérgico escalonado con doxazocina 1 mg cada $12 \mathrm{~h}$ y propanolol $10 \mathrm{mg}$ cada $8 \mathrm{~h}$. En la Tabla 3 se muestran los cambios en diversas variables durante la preparación preoperatoria, que demostraron la expansión del volumen.

La adrenalectomía izquierda se realizó 14 días después, sin presentar complicaciones hemodinámicas significativas. El postoperatorio fue favorable y a las $24 \mathrm{~h}$ presentaba presiones arteriales de

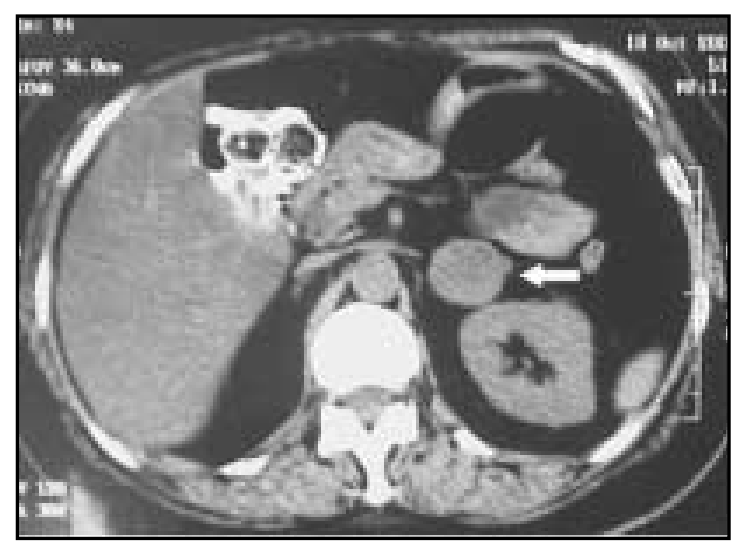

FIGURA 1. TAC de abdomen y pelvis sin contraste con nódulo suprarrenal izquierdo.

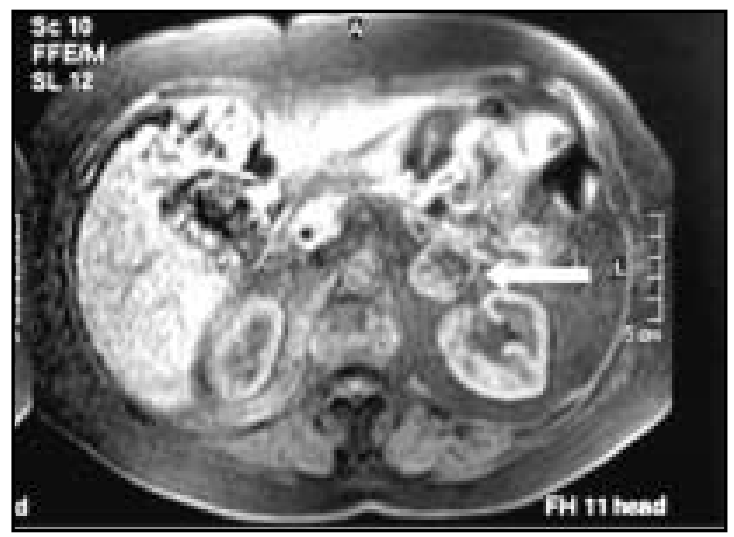

FIgURA 2. Resonancia nuclear magnética con hiperintensidad en $\mathrm{T} 2$. 
Tabla 3. Cambios durante el preoperatorio

\begin{tabular}{|lrr|}
\hline Variable & Inicio & $\begin{array}{r}\text { Pre-operatorio } \\
\text { inmediato }\end{array}$ \\
\hline Peso & $79,00 \mathrm{~kg}$ & $83,500 \mathrm{~kg}$ \\
Presión arterial de pie & $70 / 30 \mathrm{mmHg}$ & $100 / 60 \mathrm{mmHg}$ \\
Frecuencia cardíaca de pie & $130 \mathrm{por} \mathrm{min}$ & $93 \mathrm{por} \mathrm{min}$ \\
Hematocrito & $46 \%$ & $34 \%$ \\
BUN & $30 \mathrm{mg} / \mathrm{dl}$ & $9 \mathrm{mg} / \mathrm{dl}$ \\
Acido úrico & $7 \mathrm{mg} / \mathrm{dl}$ & $3,9 \mathrm{mg} / \mathrm{dl}$ \\
Albúmina & $4,5 \mathrm{gr} / \mathrm{dl}$ & $3,4 \mathrm{gr} / \mathrm{dl}$ \\
Sodio & $146 \mathrm{mEq} / \mathrm{L}$ & $136 \mathrm{mEq} / \mathrm{L}$ \\
\hline
\end{tabular}

120/70 mmHg, con frecuencia cardíaca de 70 por min. Fue dada de alta 5 días después, sin medicamentos antihipertensivos.

\section{DisCUSIÓN}

En la normotensión postural participan múltiples factores, como el funcionamiento cardíaco, volumen sanguíneo, estimulación simpática de las arteriolas periféricas y esplácnicas, reflejos originados en los barorreceptores, reflejo venoarteriolar, sistema renina-angiotensina y la acción propulsora de los músculos esqueléticos de las extremidades inferiores. Sin embargo, las fases iniciales de la ortostasis, primeros 1 a $2 \mathrm{~min}$, dependen primordialmente de la volemia intratorácica y de la vasoconstricción arteriolar y venosa. En sujetos sanos no se produce un cambio significativo en la presión sistólica ni diastólica frente al cambio postural, más aún la presión diastólica tiende a aumentar. Por otro lado, se define hipotensión ortostática al descenso $\geq 20$ $\mathrm{mmHg}$ de la presión sistólica $0 \geq 10 \mathrm{mmHg}$ de la presión diastólica en los primeros $3 \mathrm{~min}$ del cambio postural. Por lo tanto, el desarrollo de hipotensión ortostática se debe al trastorno de alguno de los factores o mecanismos mencionados previamente ${ }^{5}$.

La hipotensión ortostática se ha descrito en un porcentaje de hasta $40 \%$ de los sujetos portadores de feocromocitoma, manifestándose desde descensos leves de la presión arterial en posición de pie, pasando por una prueba de tilt positiva, hasta cuadros de colapso cardiovascular ${ }^{6}$.

Con la única excepción de hipertensos diabéticos de larga data, esta manifestación es altamente sugerente de este diagnóstico en un paciente hipertenso no tratado como ya fue descrito en 1947 por Bruce ${ }^{7}$. La diferenciación con trastornos que suelen acompañarse de hipertensión e hipotensión ortostática como la falla de los barorreflejos y la insuficiencia autonómica generalizada, puede realizarse, ya que en el feocromocitoma la hipotensión ortostática se acompaña de taquicardia y estos pacientes tienen niveles de catecolaminas plasmáticas y urinarias de $24 \mathrm{~h}$ elevados, tanto en sus momentos de hipertensión como de hipotensión, y que no disminuyen con clonidina. Además en el feocromocitoma, su aparición no tiene relación a intervenciones médicas o quirúrgicas en los territorios de los nervios glosofaríngeos o vagos, como ocurre en la falla de los barorreceptores $^{8}$.

Por otro lado, la hipertensión supina asociada a hipotensión ortostática del adulto mayor 0 diabético de larga data, se debe a varios factores como una marcada reducción de la distensibilidad de los grandes vasos arteriales, asociada a depleción parcial de volumen intravascular, acentuación de la estasia venosa en las extremidades inferiores (venous pooling) y grados variables de disfunción de barorreceptores. Estos pacientes tienen niveles normales o bajos de catecolaminas en posición supina y no desarrollan una taquicardia compensadora frente al descenso de presión en posición de pie ${ }^{9}$.

Aunque esta manifestación de feocromocitoma fue tempranamente descrita, sus mecanismos fisiopatológicos no están aún totalmente dilucidados. Los mecanismos propuestos han sido:

a) Hipovolemia: que se desarrolla a consecuencia de la intensa vasoconstricción que desplaza volumen desde el intravascular al extravascular, que no es compensada por los mecanismos retenedores de sodio $^{10}$, y por el estado hipercatabólico de estos sujetos. Aunque se ha demostrado hipovolemia en un número significativo de los pacientes con feocromocitoma e hipotensión, no todos presentan hipovolemia y no todos aquellos con hipovolemia han presentado esta manifestación ${ }^{3}$. 
b) Secreción de epinefrina: existen feocromocitomas que secretan epinefrina como catecolamina predominante, manifestándose el efecto vasodilatador sobre los receptores $\beta$-adrenérgicos en los vasos sanguíneos de músculos esqueléticos ${ }^{10}$. Sin embargo, sólo una minoría de los feocromocitomas tienen este patrón de secreción catecolaminérgica.

c) Desensibilización de los receptores adrenérgicos: debido a un fenómeno de down-regulation de estos receptores por las altas concentraciones de catecolaminas. Ya que la secreción tumoral es episódica, existirían períodos de una respuesta insuficiente para mantener la normotensión ortostática. En relación a este fenómeno, se ha descrito en estos pacientes una respuesta suprimida en la fase IV de la maniobra de Valsalva, cuya respuesta depende de la activación de $\alpha$ y $\beta$-receptores. En contraste con ello, se ha demostrado que en estos pacientes se produce una elevación de las catecolaminas plasmáticas frente a cambios posturales. Interesantemente, es ampliamente conocido que no existe correlación entre los valores de catecolaminas plasmáticas y los valores de presión arterial, lo que puede fácilmente observarse por la inmediata caída de la presión arterial después de la extirpación de un tumor único, persistiendo niveles plasmáticos elevados de estas hormonas por varios días ${ }^{11}$.

d) Alteración del sistema simpático: en que se produciría una respuesta tardía frente a la señal de los barorreceptores, basado en que se ha demostrado en algunos sujetos con esta entidad una disminución de la velocidad de conducción nerviosa a nivel simpática ${ }^{12}$. En aquellos que desarrollan descenso de la presión en posición de pie, la presión sistólica disminuye pero la presión diastólica no cambia significativamente, reduciéndose el débito cardíaco, en ausencia de vasoconstricción compensatoria $^{3}$.
En síntesis, no todos los sujetos con feocromocitoma presentan un mismo y claro mecanismo de hipotensión ortostática, participando la volemia 0 el sistema simpático como los factores primordiales.

En nuestra paciente, planteamos que el mecanismo fundamental involucrado fue la hipovolemia, basado en su examen físico y en los niveles de BUN, creatinina, relación BUN/creatinina, uricemia y proteinemia. Más aún, por los cambios observados con el aporte abundante de sal y agua en el período preoperatorio tanto en los exámenes sanguíneos mencionados y en el peso corporal, concomitantes con la desaparición de la hipotensión postural.

En concordancia con lo observado en el período preoperatorio y postoperatorio inmediato, la corrección de la hipovolemia es mandataria previo a la intervención quirúrgica, ya que la morbilidad y mortalidad de esta cirugía está dada en la actualidad por hipotensión arterial inmanejable y no por la hipertensión, debido a los avances anestésicos y quirúrgicos ${ }^{4}$. La corrección de la hipotensión ortostática es un excelente indicador para evaluar la efectividad del tratamiento preoperatorio y definir el momento de la cirugía ${ }^{13}$.

Interesantemente en esta paciente, los cambios hemodinámicos y metabólicos se expresaron notoriamente después del inicio de los antidepresivos tricíclicos, indicados por las alteraciones del ánimo, frecuentes en el feocromocitoma, y en ella interpretados como síntomas depresivos. La acentuación de los síntomas del feocromocitoma se debe al bloqueo de la recaptación de noradrenalina desde las terminales nerviosas, lo que ocasiona una marcada elevación de los niveles plasmáticos de noradrenalina. Debe tenerse presente que la aparición o agravamiento de una hipertensión en asociación a estos agentes farmacológicos, debe hacer sospechar interacciones farmacológicas o la presencia de un feocromocitoma ${ }^{14}$. 


\section{REFERENCIAS}

1. Manger W, GifFord R. The Clinical and experimental Pheochromocytoma. Second edition. Blackwell Science, Malden MA, 1998.

2. WeRBEL SS, OBER KP. Pheochromocyotoma: update on diagnosis, localization and management. Med Clin North Am 1995; 79: 131-53.

3. BRAVO E. Pheochromocytoma: new concepts and future trends. Kidney Int 1991; 40: 544-56.

4. Bravo E, Tagle R. Pheochromocytoma: State of the art and future prospects. Endocrine Reviews. 2003; 24: 539-53.

5. Mathias CJ. Orthostatic hypotension: causes, mechanisms, and influencing factors. Neurology 1995: 45 (4 Suppl 5): S6-11.

6. Bergland BE. Pheochromocytoma presenting as shock. Am J Emerg Med 1989; 7: 44-8.

7. BRucE GM. The ocular fundus in pheochromocytoma of adrenal gland: report of three cases. Trans Am Ophtalmol 1947; 45: 201-28.

8. MANGeR W. Baroreflex failure a diagnostic challenge. NEJM 1993; 329: 1494-5.

9. Schutzman J, Jaeger F, Maloney J, Fouad-Tarazi F. Head up tilt and hemodynamic changes during orthostatic hypotension in patients with supine hypertension. JACC 1994; 24: 454-61.

10. Dustan HP, Tarazi RC, Bravo EL, Dart RA. Plasma and extracellular fluid volumes in hypertension. Circulation Res 1973; 32 (Suppl 1): 73-83.

11. Bravo E. Evolving concepts in the pathophysiology, diagnosis and treatment of pheochromocytoma. Endocrine Reviews. 1994; 15: 365-8.

12. Bravo E, Tarazi RC, GifFord RW, Stewart DH. Cinculating and urinary catecholamines in pheochromocytoma. Diagnostic and pathophysiologic implications. N Engl J Med 1979; 301: 682-6.

13. MunaKata M, Aihara A, Imai $Y$, Noshiro T, Ito $S$, Yoshinaga K. Altered sympathetic and vagal modulations of the cardiovascular system in patients with pheochromocytoma: their relations to orthostatic hypotension. Am J Hypertens 1999; 12: 57280.

14. Furino A, Dambrosio M, Fiore $T$, De Biasi $S$. Difference between arterial pressure in clinoorthostatism. A method for evaluating preparation for pheochromocytoma. Minerva Anestesiologica 1989; 55: 17-22.

15. Kaufmann JS. Pheochromocytoma and tricyclic antidepressant. JAMA 1974; 229: 1282. 